\title{
Current laboratory and clinical practices in reporting and interpreting anti-nuclear antibody indirect immunofluorescence (ANA IIF) patterns: results of an international survey
}

Lieve Van Hoovels ${ }^{1,2^{*}} \oplus$, Sylvia Broeders ${ }^{3}$, Edward K. L. Chan ${ }^{4}$, Luis Andrade $^{5}$, Wilson de Melo Cruvinel ${ }^{6}$, Jan Damoiseaux ${ }^{7}$, Markku Viander ${ }^{8}$, Manfred Herold ${ }^{9}$, Wim Coucke ${ }^{3}$, Ingmar Heijnen ${ }^{10}$, Dimitrios Bogdanos ${ }^{11}$, Jaime Calvo-Alén ${ }^{12}$, Catharina Eriksson ${ }^{13}$, Ana Kozmar ${ }^{14}$, Liisa Kuhi ${ }^{15}$, Carolien Bonroy ${ }^{16,17}$, Bernard Lauwerys ${ }^{18,19}$, Sofie Schouwers ${ }^{20}$, Laurence Lutteri ${ }^{21}$, Martine Vercammen ${ }^{22,23}$, Miroslav Mayer ${ }^{24}$, Dina Patel ${ }^{25}$, William Egner ${ }^{25}$, Kari Puolakka ${ }^{26}$, Andrea Tesija-Kuna ${ }^{14}$, Yehuda Shoenfeld ${ }^{27,28}$, Maria José Rego de Sousa ${ }^{29}$, Marcos Lopez Hoyos ${ }^{30}$, Antonella Radice ${ }^{31}$ and Xavier Bossuyt ${ }^{2,32}$

\begin{abstract}
Background: The International Consensus on Antinuclear Antibody (ANA) Patterns (ICAP) has recently proposed nomenclature in order to harmonize ANA indirect immunofluorescence (IIF) pattern reporting. ICAP distinguishes competent-level from expert-level patterns. A survey was organized to evaluate reporting, familiarity, and considered clinical value of ANA IIF patterns.
\end{abstract}

Methods: Two surveys were distributed by European Autoimmunity Standardization Initiative (EASI) working groups, the International Consensus on ANA Patterns (ICAP) and UK NEQAS to laboratory professionals and clinicians.

Results: 438 laboratory professionals and 248 clinicians from 67 countries responded. Except for dense fine speckled (DFS), the nuclear competent patterns were reported by $>85 \%$ of the laboratories. Except for rods and rings, the cytoplasmic competent patterns were reported by $>72 \%$ of laboratories.

Cytoplasmic IIF staining was considered ANA positive by $55 \%$ of clinicians and $62 \%$ of laboratory professionals, with geographical and expertise-related differences.

Quantification of fluorescence intensity was considered clinically relevant for nuclear patterns, but less so for cytoplasmic and mitotic patterns. Combining IIF with specific extractable nuclear antigens (ENA)/dsDNA antibody testing was considered most informative.

Of the nuclear competent patterns, the centromere and homogeneous pattern obtained the highest scores for clinical relevance and the DFS pattern the lowest. Of the cytoplasmic patterns, the reticular/mitochondria-like pattern obtained the highest scores for clinical relevance and the polar/Golgi-like and rods and rings patterns the lowest.

\footnotetext{
*Correspondence: Lieve.Van.Hoovels@olvz-aalst.be

${ }^{1}$ Department of Laboratory Medicine, OLV Hospital, Aalst, Belgium

Full list of author information is available at the end of the article
}

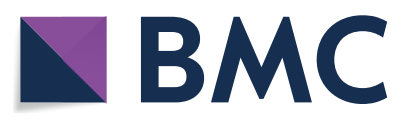

(c) The Author(s) 2020. This article is licensed under a Creative Commons Attribution 4.0 International License, which permits use, sharing, adaptation, distribution and reproduction in any medium or format, as long as you give appropriate credit to the original author(s) and the source, provide a link to the Creative Commons licence, and indicate if changes were made. The images or other third party material in this article are included in the article's Creative Commons licence, unless indicated otherwise in a credit line to the material. If material is not included in the article's Creative Commons licence and your intended use is not permitted by statutory regulation or exceeds the permitted use, you will need to obtain permission directly from the copyright holder. To view a copy of this licence, visit http://creativeco mmons.org/licenses/by/4.0/. 
Conclusion: This survey confirms that the major nuclear and cytoplasmic ANA IIF patterns are considered clinically important. There is no unanimity on classifying DFS, rods and rings and polar/Golgi-like as a competent pattern and on reporting cytoplasmic patterns as ANA IIF positive.

Keywords: Antinuclear antibodies, ANA patterns, Indirect immunofluorescence, ICAP

\section{Keymessages}

We report on an international survey on Antinuclear Antibody (ANA) indirect immunofluorescence (IIF) pattern reporting.

Both clinicians and laboratory professionals consider the major nuclear and cytoplasmic IIF patterns as clinically important.

There is no unanimity on reporting cytoplasmic patterns as ANA IIF positive.

\section{Background}

Indirect immunofluorescence (IIF) on HEp-2 cells is commonly used to screen for antinuclear antibodies (ANA). ANA are helpful for the diagnosis of ANA-associated rheumatic diseases (AARD) [1, 2]. In the recent classification criteria for systemic lupus erythematosus, the presence of ANA is an entry criterion [3]. Besides, ANA are a prognostic marker for uveitis in children with juvenile idiopathic arthritis [4] and ANA positivity and titers are included in the internationally-accepted, simplified criteria for the diagnosis of autoimmune hepatitis [5-7]. Finally, some ANA patterns can point towards specific diseases such as the reticular/mitochondria-like pattern in primary biliary cholangitis and the centromere pattern in systemic sclerosis [8-10]. Guidelines for ANA detection by IIF recommend to report ANA titer and pattern $[11,12]$. The higher the antibody level, the higher the likelihood for disease [13-15]. In addition, the combination of the antibody level and the antibody pattern can also provide helpful information [16].

The International Consensus on ANA Patterns (ICAP), a working group of the Autoantibody Standardization Committee (ASC) [17], has recently proposed an ordered nomenclature in order to harmonize the names and descriptions of distinct HEp-2 IIF pattern [18]. At the time of the survey (2019), 30 anticell (AC) patterns compose the ICAP classification tree [19]. The morphological description and examples of the AC patterns are accessible (in several languages) at the ICAP website (www.anapatterns.org and [20]). ICAP distinguishes patterns that should be readily recognized (competent-level) from patterns that require more experience (expert-level). This distinction is not only based on the fact that the pattern is easily recognizable, but also on its clinical relevance
[17]. According to ICAP, the following AC patterns are competent-level: nuclear homogenous, speckled, dense fine speckled (DFS), centromere, nucleolar and discrete dots and cytoplasmic fibrillary, speckled, reticular/ mitochondria-like, polar/Golgi-like and rods and rings. ICAP acknowledges that the distinction is a temporary status and is subject to feedback and commentaries from the international community [17].

In order to evaluate to which extent clinical laboratories and clinicians adhere to the ICAP recommendations and appreciate the clinical value of the IIF patterns, a survey on ANA IIF pattern reporting and interpretation was organized. The results of such surveys should guide further harmonization.

\section{Methods}

The Belgian European Autoimmunity Standardization Initiative (EASI) working group prepared two structured survey forms, one addressed to laboratory professionals, reporting ANA IIF test results, and one to clinicians, requesting ANA IIF tests and interpreting the ANA IIF result report. Both survey forms (available as Additional file 1 and Additional file 2) interrogated reporting, familiarity and clinical significance of ANA IIF patterns. During a 2 year period (06/2017-05/2019), the survey forms were distributed to laboratory professionals and rheumatologists by the EASI working groups. Besides, the survey forms were also distributed through UK NEQAS and ICAP. The specialty of the clinicians that participated through ICAP is not known.

Globally, the surveys forms were distributed in the following formats:

1. open, web-based survey (Austria, Spain, Italy, Croatia, Sweden, UK, Switzerland, Portugal, Estonia, Greece)

2. closed survey (Belgium, Finland, the Netherlands, laboratories that are subscribed to ICAP with exclusion of the countries mentioned above as they were addressed through EASI)

Statistical analysis (chi-squared test relative proportion) was performed in MEDCALC (software version 17.1., Ostend, Belgium). A $p$ value $<0.05$ was considered as statistically significant. 


\section{Results}

\section{Characteristics of the participating laboratories} and clinicians

Four hundred thirty-eight laboratory professionals and 248 clinicians (183 or $74 \%$ rheumatologists, for the other clinicians, specialty is not known) from 67 different countries worldwide responded to the surveys, of whom $358(82 \%)$ and 84 (34\%), respectively, completed the whole survey. Since the survey was also distributed through an open, web-based format we were unable to accurately estimate the response rate. Table 1 shows the geographic distribution of the respondents. Most of the respondents were from Europe $(259 / 438$ or $59 \%$ of laboratory professionals and $206 / 248$ or $83 \%$ of the clinicians). Fifty percent $(220 / 438)$ of the laboratory professionals that responded considered their laboratory as expert-level (i.e. recognize patterns that require more expertise) and $54 \%(135 / 248)$ of the clinicians worked in a tertiary hospital.

\section{Reporting of ANA IIF patterns}

Table 2 represents an overview of (i) the frequency of reporting of the various AC patterns, (ii) the estimation whether a pattern should be classified as competent, and (iii) the clinical relevance of the various AC patterns. For the clinical relevance score, 1 is the lowest and 5 the highest score.

For each question, the results per pattern, are presented as percentages of the total laboratory (competent and expert reported separately) and clinician respondents. The competent patterns are highlighted in bold. The reported range represents the minimum and maximum number of responders to the specific questions posed.

The competent-level patterns centromere (AC-3), homogeneous $(\mathrm{AC}-1)$, speckled $(\mathrm{AC}-2,4,5)$ and nucleolar $(\mathrm{AC}-8,9,10)$ patterns were used in ANA IIF test result reporting by $>90 \%$ of competent-level and expert-level laboratories (Table 2). The nuclear dot patterns (AC-6,7) were reported by $85 \%$ of the laboratories and the DFS pattern (AC-2) by $62 \%$ of the laboratories. Clinicians reported comparable (or somewhat lower) frequencies of being acquainted with those patterns (Table 2).

Cytoplasmic AC patterns were less frequently used in ANA IIF test result reporting than nuclear AC patterns. Among the competent cytoplasmic patterns (fibrillar (AC-15,16,17), speckled (AC-18,19,20), reticular/ mitochondria-like (AC-21), polar/Golgi-like (AC-22), rods and rings (AC-23), the rods and rings pattern ( $\mathrm{AC}-23)$ was the pattern that was least used (63\% of the laboratories) (Table 2).

The competent-level DFS pattern (AC-2) and rods and rings pattern $(\mathrm{AC}-23)$ were significantly $(\mathrm{p}<0.0001)$ more used by expert-level laboratories than by competent-level laboratories (76\% versus $48 \%$ for DFS and $73 \%$ versus $53 \%$ for rods and rings). Moreover, the DFS pattern was less reported by laboratories in North-America (44\%) and Europe (57\%) than by laboratories in the other continents (Table 3).

Table 1 Geographic distribution of the respondents with information on laboratories and clinicians showing (i) the geographic distribution of the respondents, (ii) the classification of laboratories as competent or expert and (iii) the clinical setting of the clinicians (N.a.: not applicable; Total completed: number of respondents that completed the whole survey)

\begin{tabular}{|c|c|c|c|c|c|c|c|c|c|}
\hline \multirow[t]{2}{*}{ Continent } & \multicolumn{3}{|c|}{ Laboratory professionals } & \multirow[b]{2}{*}{ Total } & \multicolumn{5}{|l|}{ Clinicians } \\
\hline & Competent & Expert & n.a & & Primary setting & Secundary setting & Tertiary setting & n.a & Total \\
\hline Africa & 6 & 4 & & 10 & & & & & \\
\hline Asia & 33 & 27 & 1 & 61 & & 2 & 11 & 1 & 14 \\
\hline Australia & 1 & 5 & & 6 & & & 2 & & 2 \\
\hline Europe & 121 & 134 & 4 & 259 & 49 & 47 & 107 & 3 & 206 \\
\hline North-America & 17 & 19 & 1 & 37 & 3 & 1 & 5 & 1 & 10 \\
\hline South-America & 30 & 31 & 3 & 64 & 1 & 4 & 10 & & 15 \\
\hline (Not assigned) & 1 & & & 1 & 1 & & & & 1 \\
\hline Total & 209 (47.7\%) & $220(50.2 \%)$ & 9 & 438 & $54(21.8 \%)$ & $54(21.8 \%)$ & 135 (54.4\%) & 5 & 248 \\
\hline Total completed & 166 & 186 & 6 & 358 & 11 & 11 & 60 & 2 & 84 \\
\hline
\end{tabular}

Countries of origin of laboratory experts: Africa: Algeria, Egypt, Morocco, Saudi-Arabia, South-Africa, Tunisia; Asia: Azerbaijan, China, Hong Kong, India, Indonesia, Iran, Israel, Korea, Lebanon, Malaysia, Pakistan, Taiwan, Thailand, United Arab Emirates; Europe: Austria, Belgium, Bosnia and Herzegovina, Bulgaria, Croatia, Czech Republic, Denmark, Estonia, Finland, France, Germany, Greece, Hungary, Italy, Kazakhstan, Lithuania, Malta, Netherlands, Norway, Poland, Portugal, Romania, Serbia, Slovakia, Spain, Sweden, Switzerland, Turkey, United Kingdom; North-America: Canada, Guatemala, Mexico, Nicaragua, Panama, United States of America; South-America: Argentina, Bolivia, Brazil, Chile, Colombia, Costa Rica, Ecuador, Peru, Uruguay, Venezuela

Countries of origin of clinicians: Asia: China, India, Japan, Korea, Malaysia, Taiwan; Europe: Austria, Belgium, Croatia, Cyprus, Estonia, Finland, Greece, Hungary, Italy, Lithuania, Netherlands, Norway, Poland, Serbia, Slovakia, Spain, Sweden, Turkey, United Kingdom; North-America: Canada, Mexico, Dominican Republic, United States of America; South-America: Argentina, Brazil, Chile, Peru 


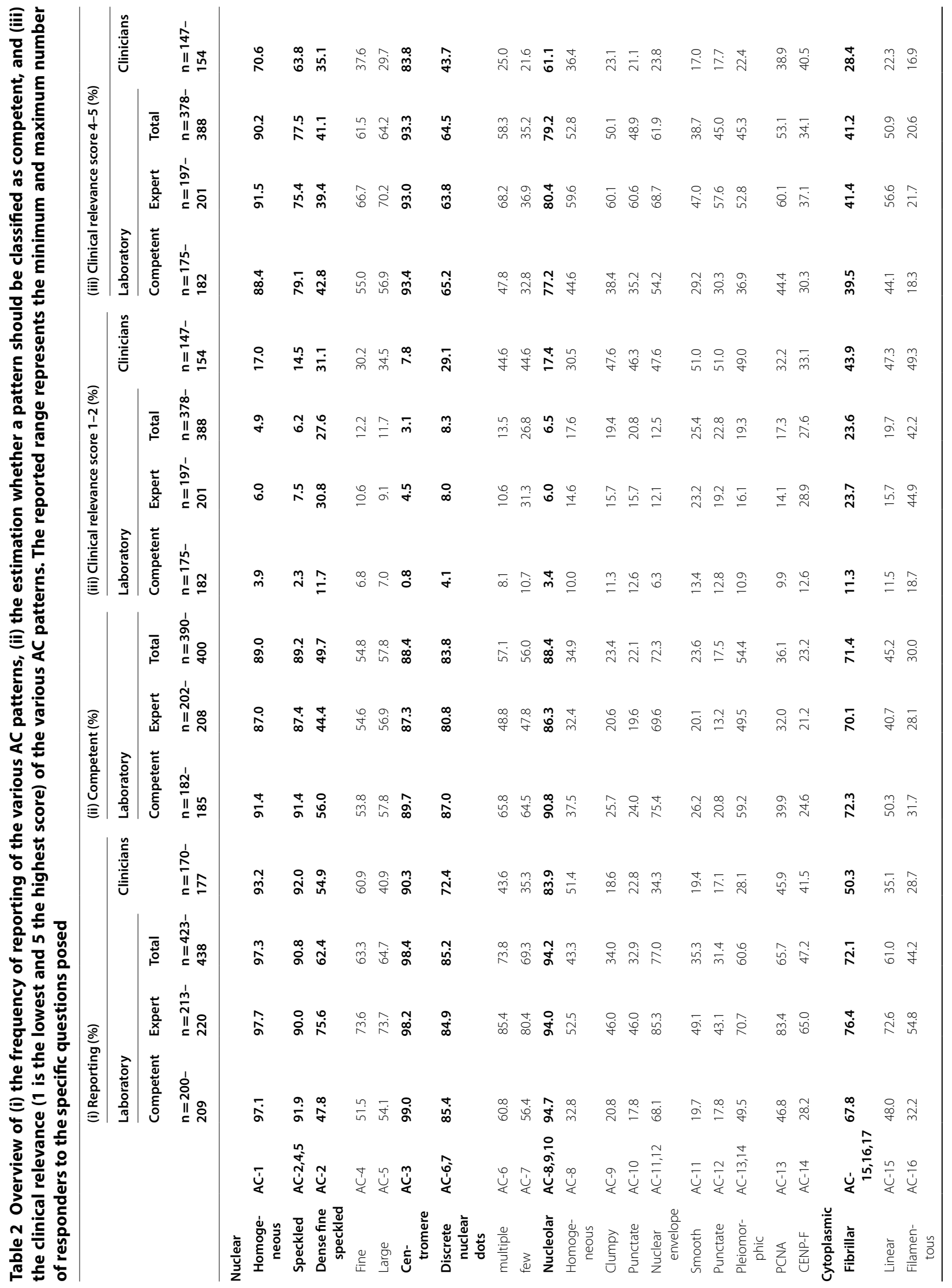




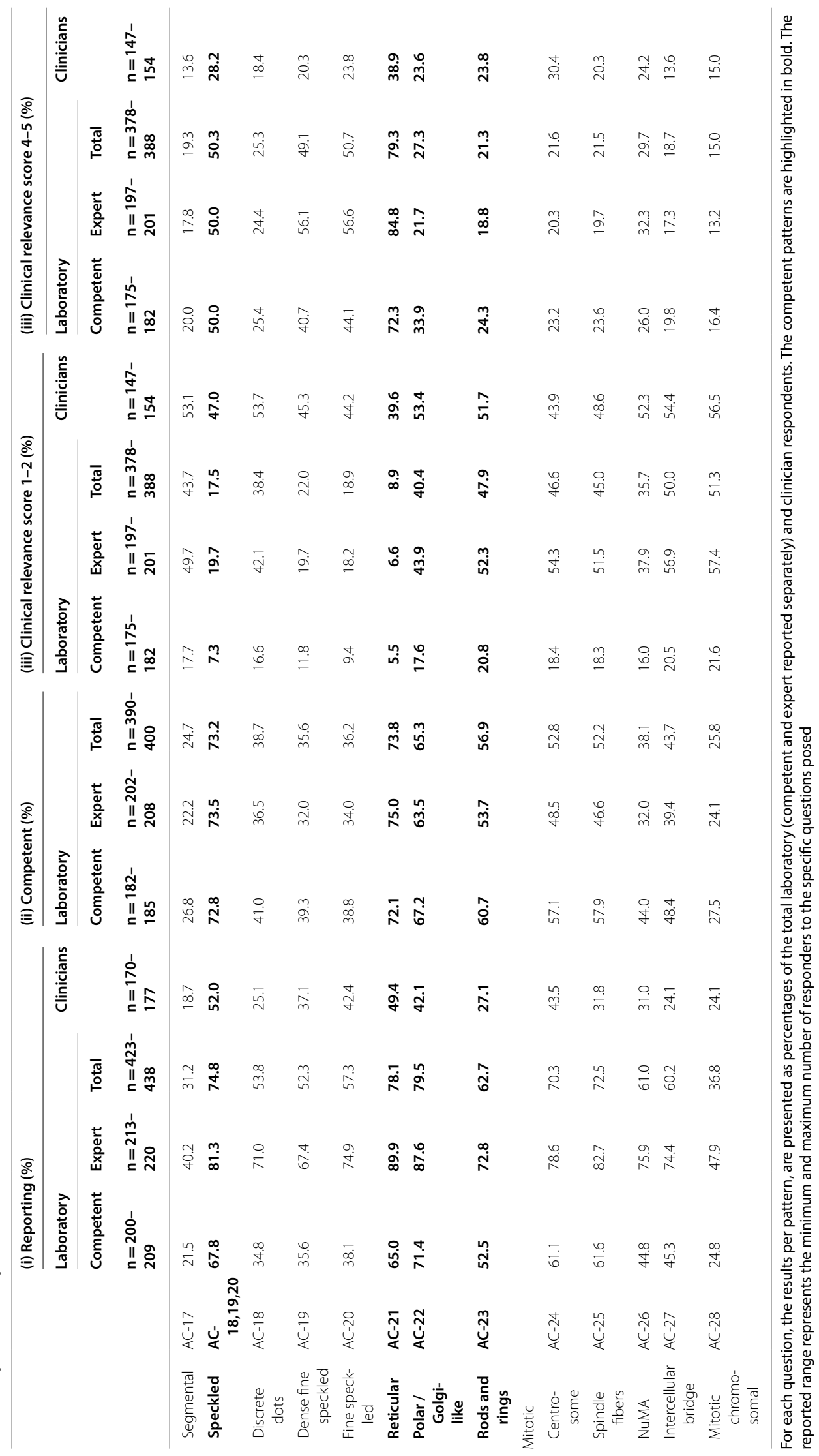


Table 3 Demographic differences in reporting the dense fine speckled (AC-2) and the rods and rings pattern (AC-23), with results shown and presented as percentage (\%) of the total responders $\%$ per geographic continent

a) Dense fine speckled (AC-2)

\begin{tabular}{|c|c|c|c|c|c|c|c|c|}
\hline \multirow[b]{3}{*}{ Continent } & \multicolumn{6}{|c|}{ Laboratory professionals } & \multicolumn{2}{|c|}{ Clinicians } \\
\hline & \multicolumn{2}{|c|}{ Total } & \multicolumn{2}{|c|}{ Competent } & \multicolumn{2}{|c|}{ Expert } & \multirow[b]{2}{*}{$\mathrm{n}$} & \multirow[b]{2}{*}{ Reporting (\%) } \\
\hline & $n$ & Reporting (\%) & $\mathrm{n}$ & Reporting (\%) & $\mathrm{n}$ & Reporting (\%) & & \\
\hline Africa & 8 & $6(75.0 \%)^{\mathrm{a}}$ & 5 & $3(60.0 \%)$ & 3 & $3(100 \%)$ & 0 & n.a \\
\hline Asia & 59 & $46(78.0 \%)^{b}$ & 33 & $22(66.7 \%)$ & 26 & $24(92.3 \%)$ & 9 & $5(55.6 \%)$ \\
\hline Australia & 6 & $5(83.3 \%)^{c}$ & 1 & $0(0.0 \%)$ & 5 & $5(100 \%)$ & 2 & $1(50.0 \%)$ \\
\hline Europe & 251 & $143(57.0 \%)$ & 118 & $48(40.7 \%)$ & 133 & $95(71.4 \%)$ & 143 & $78(54.5 \%)$ \\
\hline North Amercia & 36 & $16(44.4 \%)$ & 17 & $7(41.2 \%)$ & 19 & $9(47.4 \%)$ & 7 & $2(28.6 \%)$ \\
\hline South America & 61 & $46(75.4 \%)^{d}$ & 30 & $18(60.0 \%)$ & 31 & $28(90.3 \%)$ & 11 & $8(72.7 \%)$ \\
\hline Total & 421 & $262(62.2 \%)$ & 204 & $98(48.0 \%)$ & 217 & 164 (75.6\%) & 172 & $94(54.7 \%)$ \\
\hline
\end{tabular}

b) Rods and rings (AC-23)

\begin{tabular}{|c|c|c|c|c|c|c|c|c|}
\hline \multirow[b]{3}{*}{ Continent } & \multicolumn{6}{|c|}{ Laboratory } & \multicolumn{2}{|c|}{ Clinicians } \\
\hline & \multicolumn{2}{|c|}{ Total } & \multicolumn{2}{|c|}{ Competent } & \multicolumn{2}{|c|}{ Expert } & \multirow[b]{2}{*}{$\mathbf{n}$} & \multirow[b]{2}{*}{ Reporting (\%) } \\
\hline & $n$ & Reporting (\%) & $n$ & Reporting (\%) & $n$ & Reporting (\%) & & \\
\hline Africa & 10 & $8(80.0 \%)$ & 6 & $5(83.3 \%)$ & 4 & $3(75.0 \%)$ & 0 & n.a \\
\hline Asia & 59 & 45 (76.3\%) & 33 & $23(69.7 \%)$ & 26 & $22(84.6 \%)$ & 9 & $3(33.3 \%)$ \\
\hline Australia & 5 & $1(20.0 \%)$ & 1 & $0(0.0 \%)$ & 4 & $1(25.0 \%)$ & 2 & $0(0.0 \%)$ \\
\hline Europe & 247 & 149 (60.3\%) & 116 & 55 (47.4\%) & 131 & 94 (71.8\%) & 140 & 33 (23.6\%) \\
\hline North Amercia & 36 & $22(61.1 \%)$ & 17 & $8(47.1 \%)$ & 19 & $14(73.7 \%)$ & 7 & $2(28.6 \%)$ \\
\hline South America & 59 & $36(61.0 \%)$ & 30 & 15 (50.0\%) & 29 & $21(72.4 \%)$ & 11 & $3(27.3 \%)$ \\
\hline Total & 416 & $261(62.7 \%)$ & 203 & 106 (52.2\%) & 213 & 155 (72.8\%) & 169 & $41(24.3 \%)$ \\
\hline
\end{tabular}

a $p=0.1217$ versus North-America and $p=0.3115$ versus Europe

b $p=0.0009$ versus North-America and $p=0.0030$ versus Europe

c $p=0.0813$ versus North-America and $p=0.1985$ versus Europe

d $p=0.0022$ versus North-America and $p=0.0084$ versus Europe

N.a. not applicable

\section{Distinction between competent and expert patterns}

Laboratory professionals were also interrogated whether they would classify a pattern as competent or expert-level. For most of the nuclear patterns there was a good agreement (84-89\%) between the ICAP classification and the provided responses, except for the DFS pattern. Only $50 \%$ of the respondents would classify this pattern as competent (Table 2). Of interest, $72 \%$ of the respondents considered the nuclear envelope pattern a competent pattern rather than an expert pattern.

For the cytoplasmic patterns considered competentlevel by ICAP, $71 \%-74 \%$ of the respondents consider the fibrillary, speckled and reticular/mitochondrialike pattern a competent pattern, 65\% considered the polar/Golgi-like pattern a competent pattern and $57 \%$ considered the rods and rings pattern a competent pattern (Table 2).

Are cytoplasmic patterns considered ANA positive?

Sixty-one percent of the clinicians $(n=105)$ and $54 \%$ of the laboratory professionals $(\mathrm{n}=346)$ considered cytoplasmic HEp-2 cell IIF staining as ANA IIF positive. There were more expert-level laboratory professionals (61\%) than competent-level laboratory professionals (46\%) that considered cytoplasmic patterns as ANA positive $(\mathrm{p}=0.0062)$. The fraction of laboratory professionals that considered cytoplasmic ANA patterns as ANA positive was higher in non-European countries (63\%) than in European countries $(48 \%)(\mathrm{p}=0.0075)$ (Table 4a). However, within Europe, differences between countries were observed. In Austria (15\%; $n=2 / 13)$, the Netherlands $(12 \% ; n=3 / 25)$, Sweden $(13 \% ; n=1 / 8)$, Switzerland $(29 \%$; 
Table 4 Geographic variation in considering cytoplasmic ANA IIF patterns as ANA positive

a) The results are presented as absolute numbers and in percentage (\%) of the responders per geographic continent

\begin{tabular}{|c|c|c|c|c|c|c|}
\hline \multirow[b]{2}{*}{ Continent } & \multicolumn{2}{|l|}{ Total } & \multicolumn{2}{|c|}{ Competent } & \multicolumn{2}{|c|}{ Expert } \\
\hline & $n$ & Reporting (\%) & $n$ & Reporting (\%) & $\bar{n}$ & Reporting (\%) \\
\hline Africa & 5 & $3(60.0 \%)$ & 4 & $2(50.0 \%)$ & 1 & $1(100.0 \%)$ \\
\hline Asia & 48 & $32(66.7 \%)$ & 26 & $16(61.5 \%)$ & 22 & $16(72.7 \%)$ \\
\hline Australia & 5 & $0(0.0 \%)$ & 1 & $0(0.0 \%)$ & 4 & $0(0.0 \%)$ \\
\hline Europe & 211 & $102(48.3 \%)$ & 98 & $40(40.8 \%)$ & 113 & 62 (54.9\%) \\
\hline North Amercia & 28 & $18(64.3 \%)$ & 12 & $6(50.0 \%)$ & 16 & $12(75 \%)$ \\
\hline South America & 49 & $32(65.3 \%)$ & 23 & $12(52.2 \%)$ & 26 & 20 (76.9\%) \\
\hline Total & $346^{\mathrm{a}}$ & 187 (54.1\%) & 164 & $76(46.3 \%)$ & 182 & 111 (61.0\%) \\
\hline
\end{tabular}

b) The results are presented as percentage (\%) of the total responders per participating European country

\begin{tabular}{|c|c|c|c|c|c|c|}
\hline \multirow[b]{2}{*}{ Country } & \multicolumn{2}{|c|}{ Total } & \multicolumn{2}{|c|}{ Competent } & \multicolumn{2}{|c|}{ Expert } \\
\hline & $\mathbf{n}$ & ANA positive (\%) & $\mathbf{n}$ & ANA positive (\%) & $n$ & ANA positive (\%) \\
\hline Austria & 13 & $2(15.4 \%)$ & 3 & $0(0.0 \%)$ & 10 & $2(20.0 \%)$ \\
\hline Belgium & 34 & $22(64.7 \%)$ & 25 & $13(52 \%)$ & 9 & $9(100.0 \%)$ \\
\hline Bosnia and Herzegovina & 1 & $1(100.0 \%)$ & 1 & $1(100.0 \%)$ & n.a & n.a \\
\hline Bulgaria & 1 & $0(0.0 \%)$ & n.a & n.a & 1 & $0(0.0 \%)$ \\
\hline Croatia & 6 & $3(50.0 \%)$ & 2 & $1(50.0 \%)$ & 4 & $2(50.0 \%)$ \\
\hline Czech Republic & 1 & $0(0.0 \%)$ & 1 & $0(0.0 \%)$ & n.a & n.a \\
\hline Denmark & 1 & $0(0.0 \%)$ & 1 & $0(0.0 \%)$ & n.a & n.a \\
\hline Estonia & 5 & $5(100.0 \%)$ & 2 & $2(100.0 \%)$ & 3 & $3(100.0 \%)$ \\
\hline Finland & 4 & $2(50.0 \%)$ & 2 & $1(50.0 \%)$ & 2 & $1(50.0 \%)$ \\
\hline France & 7 & $4(57.1 \%)$ & 4 & $2(50.0 \%)$ & 3 & $2(66.7 \%)$ \\
\hline Germany & 1 & $0(0.0 \%)$ & n.a & n.a & 1 & $0(0.0 \%)$ \\
\hline Greece & 7 & $3(42.9 \%)$ & 2 & $1(50.0 \%)$ & 5 & $2(40.0 \%)$ \\
\hline Hungary & 3 & $0(0.0 \%)$ & n.a & n.a & 3 & $0(0.0 \%)$ \\
\hline Italy & 18 & $11(61.1 \%)$ & 5 & $2(40.0 \%)$ & 13 & $9(69.2 \%)$ \\
\hline Kazakhstan & 2 & $2(100.0 \%)$ & 2 & $2(100.0 \%)$ & n.a & n.a \\
\hline Malta & 1 & $1(100.0 \%)$ & n.a & n.a & 1 & $1(100 \%)$ \\
\hline Netherlands & 25 & $3(12.0 \%)$ & 20 & $2(10.0 \%)$ & 5 & $1(20.0 \%)$ \\
\hline Norway & 1 & $0(0.0 \%)$ & n.a & n.a & 1 & $0(0.0 \%)$ \\
\hline Poland & 3 & $2(66.7 \%)$ & n.a & n.a & 3 & $2(66.7 \%)$ \\
\hline Portugal & 23 & $18(78.3 \%)$ & 8 & $6(75.0 \%)$ & 15 & $12(80.0 \%)$ \\
\hline Romania & 2 & $2(100.0 \%)$ & n.a & n.a & 2 & $2(100.0 \%)$ \\
\hline Serbia & 1 & $0(0.0 \%)$ & 1 & $0(0.0 \%)$ & n.a & n.a \\
\hline Slovakia & 1 & $1(100.0 \%)$ & n.a & n.a & 1 & $1(100.0 \%)$ \\
\hline Spain & 21 & $14(66.7 \%)$ & 8 & $5(62.5 \%)$ & 13 & $9(69.2 \%)$ \\
\hline Sweden & 8 & $1(12.5 \%)$ & 1 & $0(0.0 \%)$ & 7 & $1(14.3 \%)$ \\
\hline Switzerland & 7 & $2(28.6 \%)$ & n.a & n.a & 7 & $2(28.6 \%)$ \\
\hline Turkey & 5 & $1(20.0 \%)$ & 2 & 1 (50.0\%) & 3 & $0(0.0 \%)$ \\
\hline United Kingdom & 9 & $2(22.2 \%)$ & 9 & $2(22.2 \%)$ & 0 & $0(0.0 \%)$ \\
\hline Total & 211 & 102 (48.3\%) & 99 & $41(41.4 \%)$ & 112 & $61(54.5 \%)$ \\
\hline
\end{tabular}

${ }^{a} \mathrm{n}=6$ laboratories reported expertise level' $n$ ot applicable' and $\mathrm{n}=1$ no country

$\mathrm{n}=2 / 7)$, Turkey $(20 \% ; \mathrm{n}=1 / 5)$ and the United Kingdom $(22 \% ; n=2 / 9)$, cytoplasmic ANA IIF patterns were considered ANA positive by $<30 \%$ of laboratory professionals, while in Belgium (65\%; $n=22 / 34)$, France $(57 \%$; $\mathrm{n}=4 / 7)$, Italy $(61 \% ; \mathrm{n}=11 / 18)$, Portugal (78\%; $\mathrm{n}=18 / 23)$ and Spain $(67 \% ; n=14 / 21)$, cytoplasmic patterns are considered ANA positive by $>60 \%$ of the laboratory professionals (Table 4b). 


\section{Sub-specification of patterns}

Less than half of the clinicians and laboratory professionals found it important to sub-specify (i) nucleolar patterns into homogeneous, clumpy and speckled $($ AC- $8,9,10)$ (respectively $45 \%$ and $34 \%$ ), (ii) cytoplasmic fibrillary patterns into linear, filamentous and segmental (AC-15,16,17) (respectively $28 \%$ and $42 \%$ ) and (iii) cytoplasmic speckled patterns into discrete dots, dense fine speckled and fine speckled (AC-18,19,20) (respectively $42 \%$ and $53 \%$ ). Seventy-one percent of the clinicians and $86 \%$ of the laboratory professionals found it important to report the reticular/mitochondria-like cytoplasmic pattern (AC-21). Sixty-two percent of the laboratory professionals distinguished multiple nuclear dots from few nuclear dots (AC-6,7) (data not shown).

The most used sub-specifications in ANA IIF result reports are nuclear fine speckled AC-4 (63\%), nuclear coarse speckled AC-5 (65\%), multiple nuclear dots AC-6 (74\%) and few nuclear dots AC-7 (69\%). These sub-specifications are significantly more reported by expert-level than by competent-level laboratories $(p<0.0001$ for all sub-patterns). However, clinicians are not aware of such ANA IIF sub-specification and do not consider them clinically relevant (Table 2 ).

\section{Confirmation of IIF by specific tests}

There was no difference between competent-level and expert-level laboratories regarding follow-up testing for antibodies to extractable nuclear antigens (ENA) and to dsDNA in case of a positive ANA IIF test. A geographical difference, however, was observed (Additional file 3. Table S5). Forty-one percent of European laboratories, but only $13 \%$ of North American and 10\% of South American laboratories, systematically performed followup testing for anti-ENA antibodies and to dsDNA in case of a positive ANA IIF test or on clinical indication when ANA IIF is negative. Forty-seven percent of the NorthAmerican laboratories and $44 \%$ of the South-American ones, but only $16 \%$ of the European laboratories only performed confirmation testing when the tests were specifically requested (Additional file 3 . Table S5).

The majority of competent $(72 \% ; \mathrm{n}=165)$ and expert laboratories $(79 \% ; n=179)$ review their ANA IIF results after ENA/dsDNA confirmation testing, but only a minority would change the results (respectively $8 \%$ and $10 \%$ ) or add a comment if clinically relevant (respectively 36 and $46 \%)$.

Interpretation and clinical significance of ANA IIF patterns Both clinicians $(82 \% ; n=246)$ and laboratory professionals $(81 \% ; n=352)$ considered the combination of an ANA
IIF test result with a corresponding specific anti-ENA or anti-dsDNA test result as the most clinically relevant information.

The quantification of the nuclear ANA IIF fluorescence intensity was found clinically relevant by $79 \%$ of laboratory professionals and by $74 \%$ of clinicians (Additional file 4. Table S6). Thirty-three percent of laboratory professionals and $22 \%$ of clinicians reported that one should not titrate cytoplasmic patterns. Forty-three percent of laboratory professionals and 33\% of clinicians reported that mitotic patterns should not be titrated (Additional file 4. Table S6).

Fifty-four percent of the clinicians $(n=107)$ reported that they would take medical decisions based on ANA IIF titer, whereas $87 \%$ of the clinicians reported that they would take medical decision based on results of specific testing for anti-ENA or anti-dsDNA.

Clinicians and laboratory professionals were also interrogated on how they appraise the clinical relevance of the various ANA patterns. Overall, clinicians scored the clinical relevance of the various patterns lower than laboratory professionals (Table 2). The highest scores for clinical relevance were obtained for the centromere (AC-3) pattern and the homogeneous (AC-1) pattern. Ninety-three percent of the laboratory professionals and $84 \%$ of the clinicians scored the clinical relevance of the centromere pattern high, and $90.2 \%$ of the laboratory professionals and $70.6 \%$ of the clinicians scored the clinical relevance of the homogeneous pattern high. Seventyseven to $80 \%$ of the laboratory professionals and $61-64 \%$ of the clinicians scored the clinical relevance of the nuclear speckled pattern $(\mathrm{AC}-2,4,5)$ and nucleolar pattern $(\mathrm{AC}-8,9,10)$ high. In contrast, only $41 \%$ of laboratory professionals and $35 \%$ of clinicians scored the clinical relevance of the DFS pattern (AC-2) high.

Of the cytoplasmic patterns, the reticular/mitochondria-like pattern (AC-21) obtained the highest score for clinical relevance by laboratory professionals. The other competent cytoplasmic patterns received lower scores, with the lowest scores for polar/Golgi-like (AC-22) and rods and rings (AC-23).

The mitotic patterns obtained low scores for clinical relevance overall with $\leq 30 \%$ of the clinicians and laboratory professionals scoring these patterns as clinically relevant.

\section{Discussion}

In the current paper, we present the results of a recent international survey on the reporting and interpretation of ANA IIF results in current daily practice. The results revealed concordances but also discordances with the recommendations posed by ICAP [18]. 
The survey confirmed that there is no consensus on whether anti-cytoplasmic antibodies should be considered ANA IIF positive or negative [21, 22]. Even within Europe, there are divergences between countries, as the geographic block represented by Portugal, Spain, Italy, France and Belgium tend to report exclusive cytoplasmic reactivity as a positive test, whereas countries out of this axis only report nuclear reactivity as a positive test. ANA is the common clinical and laboratory term used for more than 50 years. The term is maintained for historical reasons as well as for laboratory coding and invoicing. However, the name 'antinuclear' for the HEp-2 cell IIF test does not take into consideration that autoantibodies to cell compartments other than the nucleus (i.e. cytoplasm, mitotic) are also detected and that these antibodies may be clinically relevant [10]. Therefore, the ICAP executive board, already in their first report, advocates that in situations where there is a clear cytoplasmic or mitotic apparatus reactivity, these results need to be reported to the clinician. However, even within ICAP, the discussion on whether those patterns should be regarded as ANA IIF positive or negative has not reached consensus $[10,17,23]$. Being aware that the term 'Antinuclear antibody (ANA) test' is inappropriate, the use of the suggested alternative name, HEp-2 IIF test [10], will foster harmonization of reporting ANA IIF results.

The familiarity with ANA IIF patterns is mainly clear for the nuclear competent patterns (with the exception of DFS) and the cytoplasmic reticular/mitochondria-like antibody (AC-21) pattern, findings which are in concordance to the results of a similar recent survey performed by the American association of Medical Laboratory Immunologists [24]. Clinicians were less familiar with the cytoplasmic reticular pattern than laboratory professionals, which might be related to the fact that the survey was mainly directed to rheumatologists (and not hepatogastroenterologists). Overall, cytoplasmic and mitotic patterns are less reported by laboratories than nuclear patterns. The integration of computer-aided immunofluorescence microscopy could further improve the familiarity and consistency in pattern assignments [25, 26], although currently the existing systems are able to recognize only a small fraction of patterns.

The survey shows that not all laboratories report the DFS pattern and the rods and ring pattern and that not all expert-level laboratory professionals are convinced that these two patterns should be classified as competent.

The DFS pattern (AC-2) can vary depending on the manufacturer of the HEp-2 cell substrates $[27,28]$ and its correct identification can be challenging, resulting in a high frequency of cases misclassified as DFS pattern [28, 29]. Furthermore, the assumed clinical relevance of the DFS pattern to exclude AARD only holds if the specificity is confirmed and if it is monospecific for DFS70 [10, 27, 30]. The inclusion of anti-DFS70 antibody testing in a diagnostic algorithm or reflex testing is currently under discussion [31, 32]. It has been proposed to better define the DFS AC-2 pattern in the ICAP classification algorithm including a new pattern called 'pseudo-DFS' referring to the nuclear speckled pattern with clear staining of the metaphase plate, but without the typical features of the DFS (AC-2) pattern (i.e. a more homogeneous distribution and more uniform brightness of the nuclear speckles than in the typical DFS pattern). Distinguishing this pattern from the classical DFS pattern would be important to increase the accuracy in the interpretation of the ANA IIF test [33]. The recent availability of a reference serum with mono-reactivity to DFS70 by the Autoantibody Standardization Committee of the International Union of Immunology Societies (www.autoab.org) should help laboratories across the world to improve the ability in recognizing the DFS (AC-2) pattern [34].

In contrast to the DFS pattern, the rods and rings (AC23) pattern is easily recognizable and there is a defined clinical association (Hepatitis $\mathrm{C}$ virus infection under treatment with $\alpha$-interferon and ribavirin) [10]. However, as mentioned in the ICAP nomenclature tree (www. anapatterns.org), the AC-23 ANA IIF pattern is only detectable in selected HEp- 2 cell slides. Thus, depending on the slide source (i.e. manufacturer) used, labs will be able or unable to report this pattern. This likely explains in part the relatively low recognition rate of this pattern even in expert laboratories.

Overall, the patterns that are most frequently reported are the patterns that are considered to have the highest clinical relevance, with some exceptions such as the centrosome, Golgi and spindle fiber patterns, which are reported by circa $80 \%$ of the laboratories despite not having peculiar clinical associations. In addition, the DFS pattern is reported in less than $50 \%$ of laboratories. Considering that this pattern includes the staining of the metaphase plate, the DFS pattern can be misclassified as nuclear homogeneous pattern (AC-1), which has completely different immunological and clinical implications.

In daily routine practice, sub-specifying nucleolar (AC$8,9,10)$ patterns, cytoplasmic fibrillar patterns and cytoplasmic speckled patterns is infrequently performed, even among experts. However, clinicians do not consider such sub-classification clinically relevant (Table 2).

The highest scores for clinical relevance were obtained for the centromere (AC-3) and homogeneous $(\mathrm{AC}-1)$ pattern, followed by the nuclear speckled $(\mathrm{AC}-2,4,5)$ and cytoplasmic reticular (AC-21) patterns. Especially the centromere pattern is associated with a specific disease (limited systemic sclerosis, primary biliary cholangitis and Sjögren's syndrome) [16]. It is the only pattern that 
has an almost absolute association with a restrict set of autoantibody specificities (anti-CENP-B and/or antiCENP-A). For all other patterns, follow-up testing to identify the target antigen is mandatory, and the pattern can help to guide such follow-up testing [10].

The clinical significance of the cytoplasmic patterns as a whole was considered lower compared to nuclear patterns. Even cytoplasmic patterns such as the (dense) fine speckled patterns that are associated with clinically relevant antibodies such as the anti-synthetase antibodies, anti-SRP and anti-Rib-P are not widely recognized as clinically relevant patterns. This perception clearly indicates the need for implementing education programs in order to increase the awareness of such associations and improve the interpretation and optimal use of the ANA IIF test. The polar/Golgi-like pattern is considered a competent pattern. It is easily recognizable but its clinical association with a disease is limited [10,35-37].

The quantification of the ANA IIF fluorescence intensity was found clinically relevant for the nuclear ANA IIF patterns by a substantial fraction of clinicians $(74 \%)$ and laboratory experts (79\%). The importance of the autoantibody titer (level) is recognized by guidelines and recommendations $[1,3,11,12]$. Recent studies showed that the likelihood for AARD increases with increasing ANA IIF fluorescence intensity [13-15]. However, important variation in the detection and titration of ANAs has been reported with manual and automated methods [38-41].

Clinicians are aware of the differences between laboratories for both ANA IIF and specific ENA/dsDNA testing. A low percentage of clinicians take medical decisions based on the ANA IIF titer (54\%).

Clinicians and laboratory professionals considered that combined information on HEp-2 ANA and specific tests for anti-ENA and anti-dsDNA antibodies is most informative. This is in line with recent studies that showed that combining an immunoassay with ANA IIF adds value if the results of both tests are correctly judged in the context of the clinical manifestations of the patient [42, 43]. Furthermore, combining IIF with a solid-phase assay can assist in patient stratification, especially in case of a low-positive ANA IIF titer [42, 44, 45]. ICAP promotes to integrate the ANA IIF titer and pattern with advice for follow-up testing, taking into account the clinical presentation of the patient $[10,46]$.

\section{Conclusions}

In conclusion, this international survey confirms that the major nuclear (homogenous, speckled, centromere, nucleolar and dots) and cytoplasmic (reticular) patterns are considered clinically important. It also supports the ICAP classification in competent and expert patterns for the majority of the competent patterns, but there is no unanimity on classifying DFS, rods and rings and polar/Golgi-like as a competent pattern. Compared to nuclear patterns, cytoplasmic patterns are less reported and their clinical relevance is considered lower than the clinical relevance of nuclear patterns. Combining IIF with testing for specific antibodies is considered to be most informative. There is no unanimity among the respondents regarding the question whether a cytoplasmic pattern is ANA IIF positive.

\section{Supplementary information}

\section{Supplementary information accompanies this paper at https://doi. org/10.1186/s13317-020-00139-9.}

Additional file 1. International EASI questionnaire ANA IIF patterns for Clinicians.

Additional file 2. International EASI questionnaire ANA IIF patterns for laboratory professionals.

Additional file 3. Table S5. Demographic differences in confirming ANA IIF results with specific tests for anti-ENA and anti-dsDNA.

Additional file 4. Table S6. Overview of the fraction of respondents (laboratory professionals or clinicians) that considered it important to report the fluorescence intensity of nuclear, cytoplasmic or mitotic ANA IIF patterns.

\section{Abbreviations}

AARD: Antinuclear antibody associated rheumatic diseases; AC: Anti-cell; ANA: Antinuclear antibody; ASC: Autoantibody Standardization Committee; ICAP: The International Consensus on Antinuclear Antibody Patterns; IIF: Indirect immunofluorescence; EASI: European Autoimmunity Standardization Initiative; DFS: Dense fine speckled; ENA: Extractable nuclear antigens.

\section{Acknowledgements}

We truly thank all laboratory professionals and clinicians that participated to the surveys.

\section{Authors' contributions}

$\mathrm{LVH}, \mathrm{SB}, \mathrm{SS}$ and XB designed the study. SB distributed the survey among the participating expert organizations. The distribution among local members of the (international) organizations was performed by LA, DB, EKLC, JD, WdMC, $\mathrm{CE}, I H, M H, A K, L K, M L H, D P, A R, M J R d S$ and MV. LVH and XB drafted the manuscript. All authors read the manuscript, gave valuable comments, edited the manuscript and agreed upon the final submission. All authors read and approved the final manuscript.

\section{Funding}

Not applicable.

\section{Availability of data and materials}

The datasets used and analyzed during the current study are available from the corresponding author on reasonable request.

\section{Ethics approval and consent to participate}

Not applicable.

\section{Consent for publication}

Not applicable.

\section{Competing interest}

The authors declare that the research was conducted in the absence of any commercial or financial relationships that could be construed as a potential competing interest. 


\section{Author details}

${ }^{1}$ Department of Laboratory Medicine, OLV Hospital, Aalst, Belgium. ${ }^{2}$ Department of Microbiology and Immunology, KU Leuven, Leuven, Belgium.

${ }^{3}$ Sciensano (Formerly Scientific Institute of Public Health), Brussels, Belgium.

${ }^{4}$ Department of Oral Biology, University of Florida, Gainesville, FL, USA.

${ }^{5}$ Rheumatology Division, Universidade Federal de São Paulo, Escola Paulista de Medicina, Sao Paulo, Brazil. ${ }^{6}$ Pontifícia Universidade católica de Goiás, Goiânia, Brazil. ${ }^{7}$ Centraal Diagnostisch Laboratorium, MUMC, Maastricht, The Netherlands. ${ }^{8}$ Department of Medical Microbiology and Immunology, University of Turku, Turku, Finland. ${ }^{9}$ Rheumatology Laboratory, Department of Internal Medicine II, Medical University of Innsbruck, Innsbruck, Austria.

${ }^{10}$ Medical Immunology, Laboratory Medicine, University Hospital Basel, Basel, Switzerland. ${ }^{11}$ Department of Rheumatology and Clinical Immunology, Faculty of Medicine, School of Health Sciences, University of Thessaly, Larissa, Greece. ${ }^{12}$ Servicio de Reumatología, Hospital Universitario Araba, Vitoria, Spain. ${ }^{13}$ Department of Clinical Microbiology, Umeå University, Umeå, Sweden. ${ }^{14}$ Department of Laboratory Diagnostics, University Hospital Centre Zagreb, Zagreb, Croatia. ${ }^{15}$ Central Laboratory, East Tallin Central Hospital, Tallin, Estonia. ${ }^{16}$ Department of Internal Medicine, Ghent University, Ghent, Belgium. ${ }^{17}$ Department of Diagnostic Sciences, Ghent University, Ghent, Belgium. ${ }^{18}$ Pôle de Pathologies Rhumatismales Et systémiques, Institut de Recherche Expérimentale et Clinique, Université Catholique de Louvain, Brussels, Belgium. ${ }^{19}$ Department of Rheumatology, Cliniques Universitaires Saint-Luc, Brussels, Belgium. ${ }^{20}$ Department of Laboratory Medicine, GZA Hospitals, Antwerp, Belgium. ${ }^{21}$ Department of Clinical Chemistry, University Hospital Liège, Liège, Belgium. ${ }^{22}$ Department of Laboratory Medicine, AZ SintJan Hospital Bruges-Ostend, Bruges, Belgium. ${ }^{23}$ Research Group Reproductive Immunology and Implantation (REIM), Vrije Universiteit Brussel, Brussels, Belgium. ${ }^{24}$ Division of Clinical Immunology and Rheumatology, Department of Internal Medicine, University Hospital Centre Zagreb, Zagreb, Croatia. ${ }^{25}$ UK NEQAS Immunology, Northern General Hospital, Immunochemistry \& Allergy, Sheffield, UK. ${ }^{26}$ Department of Medicine, South Karelia Central Hospital, Lappeenranta, Finland. ${ }^{27}$ Laboratory of the Mosaic of Autoimmunity, Saint Petersburg State University, Saint-Petersburg, Russian Federation. ${ }^{28}$ Zabludowicz Center for Autoimmune Diseases, Sheba Medical Center, Affiliated to TelAviv University School of Medicine, Tel-Hashomer, Tel Aviv, Israel. ${ }^{29}$ Centro de Medicina, Laboratorial Germano de Sousa, Lisboa, Portugal. ${ }^{30}$ Servicio de Inmunología, Hospital Universitario Marqués de Valdecilla, Santander, Cantabria, Spain. ${ }^{31}$ UOC Microbiologia e Virologia, Presidio Ospedaliero San Carlo Borromeo, Milan, Italy. ${ }^{32}$ Department of Laboratory Medicine, University Hospital Leuven, Leuven, Belgium.

Received: 29 July 2020 Accepted: 29 August 2020

Published online: 23 November 2020

\section{References}

1. Solomon DH, Kavanaugh AJ, Schur PH, American College of Rheumatology Ad Hoc Committee on Immunologic Testing Guidelines. Evidencebased guidelines for the use of immunologic tests: antinuclear antibody testing. Arthritis Rheum. 2002;47:434-44.

2. Meroni PL, Schur PH. ANA screening: an old test with new recommendations. Ann Rheum Dis. 2010;69:1420-2.

3. Aringer M, Costenbader K, Daikh D, Brinks R, Mosca M, Ramsey-Goldman R, et al. 2019 European League Against Rheumatism/American College of Rheumatology classification criteria for systemic lupus erythematosus. Ann Rheum Dis. 2019;78:1151-9.

4. Angeles-Han ST, Ringold S, Beukelman T, Lovell D, Cuello CA, Becker ML, et al. 2019 American College of Rheumatology/Arthritis Foundation guideline for the screening, monitoring, and treatment of juvenile idiopathic Arthritis-Associated Uveitis. Arthritis Care Res (Hoboken). 2019;71:703-16.

5. Hennes EM, Zeniya M, Czaja AJ, Parés A, Dalekos GN, Krawitt EL, et al. Simplified criteria for the diagnosis of autoimmune hepatitis. Hepatology. 2008;48:169-76.

6. Liver EAftSot. EASL clinical practice guidelines: autoimmune hepatitis. J Hepatol. 2015;63:971-1004.

7. Mieli-Vergani G, Vergani D. Autoimmune hepatitis. Nat rev Gastroenterol Hepatol. 2011:8:320-9.
8. Bowlus CL, Gershwin ME. The diagnosis of primary biliary cirrhosis. Autoimm rev. 2014;13:441-4.

9. Liver EAftSot. EASL clinical practice guidelines: management of cholestatic liver diseases. J Hepatol. 2009;51:237-67.

10. Damoiseaux J, Andrade LEC, Carballo OG, Conrad K, Francescantonio PLC, Fritzler MJ, et al. Clinical relevance of HEp-2 indirect immunofluorescent patterns: the International Consensus on ANA patterns (ICAP) perspective. Ann Rheum Dis. 2019;78:879-89.

11. Agmon-Levin N, Damoiseaux J, Kallenberg C, Sack U, Witte T, Herold M, et al. International recommendations for the assessment of autoantibodies to cellular antigens referred to as anti-nuclear antibodies. Ann Rheum Dis. 2014;73:17-23.

12. Vergani D, Alvarez F, Bianchi FB, Cançado EL, Mackay IR, Manns MP, et al. Liver autoimmune serology: a consensus statement from the committee for autoimmune serology of the International Autoimmune Hepatitis Group. J Hepatol. 2004;41:677-83.

13. Bossuyt $X$, Cooreman S, De Baere H, Verschueren P, Westhovens R, Blockmans $D$, et al. Detection of antinuclear antibodies by automated indirect immunofluorescence analysis. CCA. 2013;415:101-6.

14. Schouwers S, Bonnet M, Verschueren P, Westhovens R, Blockmans $D$, Mariën $G$, et al. Value-added reporting of antinuclear antibody testing by automated indirect immunofluorescence analysis. CCLM. 2014;52:547-51.

15. Oyaert M, Bossuyt X, Ravelingien I, Van Hoovels L. Added value of indirect immunofluorescence intensity of automated antinuclear antibody testing in a secondary hospital setting. CCLM. 2016;54:e63-e6666.

16. Vulsteke JB, Willems $P$, Vander Cruyssen B, Vanderschueren S, Westhovens $R$, et al. Titre-specific positive predictive value of antinuclear antibody patterns. Ann Rheum Dis. 2019. https://doi.org/10.1136/ annrhuemdis-2019-216245.

17. Chan EK, Damoiseaux J, Carballo OG, Conrad K, de Melo CW, Francescantonio $\mathrm{PL}$, et al. Report of the first international consensus on standardized nomenclature of antinuclear antibody HEp-2 cell patterns 2014-2015. Front Immunol. 2015;6:412.

18. Chan EK, Damoiseaux J, de Melo CW, Carballo OG, Conrad K, Francescantonio $\mathrm{PL}$, et al. Report on the second International Consensus on ANA Pattern (ICAP) workshop in Dresden 2015. Lupus. 2016;25:797-804.

19. Andrade LEC, Klotz W, Herold M, Conrad K, Rönnelid J, Fritzler MJ, et al. International consensus on antinuclear antibody patterns: definition of the AC-29 pattern associated with antibodies to DNA topoisomerase I. CCLM. 2018;56:1783-8.

20. Damoiseaux J, Bossuyt X, Broeders S, Hamann D, de Leeuw K, Limper M, et al. International Consensus on ANA Patterns (ICAP): inbedding in het Nederlandse taalgebied. Laboratoriumgeneeskunde. 2018;1:17-26.

21. Van Hoovels L, Bossuyt X. Harmonisation of laboratory tests for rheumatic diseases: still a long way to go. Ann Rheum Dis. 2020;79:e5.

22. Infantino M, Manfredi M, Soda P, Merone M, Afeltra A, Rigon A. ANA testing in 'real life'. Ann Rheum Dis. 2020;79:e3.

23. Damoiseaux J, von Mühlen CA, La Garcia-De Torre I, Carballo OG, de Melo Cruvinel W, Francescantonio PL, et al. International consensus on ANA patterns (ICAP): the bumpy road towards a consensus on reporting ANA results. Auto Immun Highlights. 2016;7:1.

24. Peterson LK, Tebo AE, Wener MH, Copple SS, Fritzler MJ. Assessment of antinuclear antibodies by indirect immunofluorescent assay: report from a survey by the American Association of Medical Laboratory Immunologists. CCLM. 2020. https://doi.org/10.1515/cclm-2019-1262.

25. Bizzaro N, Antico A, Platzgummer S, Tonutti E, Bassetti D, Pesente F, et al. Automated antinuclear immunofluorescence antibody screening: a comparative study of six computer-aided diagnostic systems. Autoimm Rev. 2014;13:292-8.

26. van Beers JJBC, Hahn M, Fraune J, Mallet K, Krause C, Hormann W, et al. Performance analysis of automated evaluation of antinuclear antibody indirect immunofluorescent tests in a routine setting. Auto Immun Highlights. 2018;9:8.

27. Mahler M, Fritzler MJ. The clinical significance of the dense fine speckled immunofluorescence pattern on HEp-2 cells for the diagnosis of systemic autoimmune diseases. Clin Dev Immunol. 2012;2012:494356.

28. Bizzaro N, Tonutti E, Villalta D. Recognizing the dense fine speckled/lens epithelium-derived growth factor/p75 pattern on HEP-2 cells: not an easy task! Arthritis Rheum. 2011;63:4036-7. 
29. Bentow C, Fritzler MJ, Mummert E, Mahler M. Recognition of the dense fine speckled (DFS) pattern remains challenging: results from an international internet-based survey. Auto Immun Highlights. 2016;7:8.

30. Infantino M, Pregnolato F, Bentow C, Mahler M, Benucci M, Li Gobbi F, et al. Only monospecific anti-DFS70 antibodies aid in the exclusion of antinuclear antibody associated rheumatic diseases: an Italian experience. CCLM. 2019;57:1764-9.

31. Choi MY, Clarke AE, St Pierre Y, Hanly JG, Urowitz MB, Romero-Diaz J, et al. The prevalence and determinants of anti-DFS70 autoantibodies in an international inception cohort of systemic lupus erythematosus patients. Lupus. 2017;26:1051-9.

32. Bonroy C, Schouwers S, Berth M, Stubbe M, Piette Y, Hoffman I, et al. The importance of detecting anti-DFS70 in routine clinical practice: comparison of different care settings. CCLM. 2018;56:1090-9.

33. Infantino M, Shovman O, Pérez D, Grossi V, Manfredi M, Benucci M, et al. A better definition of the anti-DFS70 antibody screening by IIF methods. J immunol Methods. 2018;461:110-6.

34. Dellavance A, Baldo DC, Zheng B, Mora RA, Fritzler MJ, Hiepe F, et al. Establishment of an international autoantibody reference standard for human anti-DFS70 antibodies: proof-of-concept study for a novel Megapool strategy by pooling individual specific sera. CCLM. 2019;57:1754-63.

35. Vermeersch $P$, Bossuyt X. Prevalence and clinical significance of rare antinuclear antibody patterns. Autoimm rev. 2013;12:998-1003.

36. Vermeersch $P$, Van den Bergh K, Blockmans D, Westhovens R, Bossuyt $X$. Anti-Golgi autoantibodies are not clinically associated with systemic autoimmune diseases. Ann Rheum Dis. 2011;70:234-5.

37. Van den Bergh $K$, Vercammen $M$, Regenass $S$, Derua $R$, Vermeersch $P$, Pokreisz $\mathrm{P}$, et al. Betaine homocysteine methyl transferase 1, a novel auto-antigen associated with anti-Golgi immune reactivity. CCA. 2012;413:105-8

38. Pisetsky DS, Spencer DM, Lipsky PE, Rovin BH. Assay variation in the detection of antinuclear antibodies in the sera of patients with established SLE. Ann Rheum Dis. 2018:77:911-3.
39. Mahler M. Lack of standardisation of ANA and implications for drug development and precision medicine. Ann Rheum Dis. 2019;78:e33.

40. Van Hoovels L, Schouwers S, Van den Bremt S, Bossuyt X. Variation in antinuclear antibody detection by automated indirect immunofluorescence analysis. Ann Rheum Dis. 2018. https://doi.org/10.1136/annrheumdi s-2018-213543

41. Bogaert L, Van den Bremt S, Schouwers S, Bossuyt X, Van Hoovels L. Harmonizing by reducing inter-run variability: performance evaluation of a quality assurance program for antinuclear antibody detection by indirect immunofluorescence. CCLM. 2019;57:990-8.

42. Willems P, De Langhe E, Claessens J, Westhovens R, Van Hoeyveld E, Poesen K, et al. Screening for connective tissue disease-associated antibodies by automated immunoassay. CCLM. 2018;56:909-18.

43. Orme ME, Andalucia C, Sjölander S, Bossuyt X. A comparison of a fluorescence enzyme immunoassay versus indirect immunofluorescence for initial screening of connective tissue diseases: Systematic literature review and meta-analysis of diagnostic test accuracy studies. Best Pract Res Clin Rheumatol. 2018:32:521-34.

44. Claessens J, Belmondo T, De Langhe E, Westhovens R, Poesen K, Hüe S, et al. Solid phase assays versus automated indirect immunofluorescence for detection of antinuclear antibodies. Autoimm Rev. 2018;17:533-40.

45. Bizzaro N, Brusca I, Previtali G, Alessio MG, Massimo D, Platzgummer $\mathrm{S}$, et al. The association of solid-phase assays to immunofluorescence increases the diagnostic accuracy for ANA screening in patients with autoimmune rheumatic diseases. Autoimmun Rev. 2018;17:541-7.

46. Meroni PL, Chan EK, Damoiseaux J, Andrade LEC, Bossuyt X, Conrad K, et al. Unending story of the indirect immunofluorescence assay on HEp-2 cells: old problems and new solutions? Ann Rheum Dis. 2019;78:e46.

\section{Publisher's Note}

Springer Nature remains neutral with regard to jurisdictional claims in published maps and institutional affiliations.
Ready to submit your research? Choose BMC and benefit from:

- fast, convenient online submission

- thorough peer review by experienced researchers in your field

- rapid publication on acceptance

- support for research data, including large and complex data types

- gold Open Access which fosters wider collaboration and increased citations

- maximum visibility for your research: over $100 \mathrm{M}$ website views per year

At BMC, research is always in progress.

Learn more biomedcentral.com/submissions 